\title{
Explicit Formulation of the Solution of Hamada-Leray-Wagschal's Theorem
}

\author{
By \\ Renaud CAMALÈS*
}

\begin{abstract}
In this paper, an explicit formula of the solution of Hamada-Leray-Wagschal's theorem is given. For this, only structure's theorem of finite dimensional determination's function and linear algebra technics developped in [1] are used.
\end{abstract}

\section{$\S 1$. Introduction}

In a previous paper, the monodromy of the ramified Cauchy problem was studied [1]. This paper is a direct application of previous methods to give an explicit formulation of the solution of Hamada-Leray-Wagschal's theorem [3, 4]. First, we call back what kind of problem it is. Let $a(x, D)$ a linear differential operator of order $m$

$$
a(x, D)=\sum_{|\alpha| \leq m} a_{\alpha}(x) D^{\alpha}
$$

with holomorphic coefficients in a neighbourhood of the origin of $\mathbf{C}^{n+1}$, that is $a_{\alpha} \in \mathbf{C}\{x\}$ (where $x=\left(x_{0}, \ldots, x_{n}\right)$ is the variable of $\mathbf{C}^{n+1}$ and $x^{\prime}=$ $\left.\left(x_{1}, \ldots, x_{n}\right)\right)$. Its principal symbol will be written $g(x ; \xi)$ and we assume the hyperplane $S: x_{0}=0$ non-characteristic in the origin : this means that $g(0 ; 1,0, \ldots, 0) \neq 0$. We will note $\Omega_{0}$ an open neighbourhood of the origin of $\mathbf{C}^{n+1}$ such that all functions $a_{\alpha}$ are defined and holomorphic on $\Omega_{0}$.

Communicated by T. Kawai. Received December 3, 2003. Revised February 16, 2004. 2000 Mathematics Subject Classification(s): 32S40, 35A20, 35P15.

Key words: Ramified Cauchy Problem; Monodromy; Finite dimensional determination's functions.

Supported by the European Commission through the Research Training Network HPRNCT-1999-00118 "Geometric Analysis"

*Institut für Mathematik, Universität Potsdam, Box 601553, 14415 Potsdam, Germany. e-mail:camales@math.uni-potsdam.de or renaud_camales@yahoo.fr 
We suppose that the operator $a(x, D)$ is an operator with multiple characteristics of constant multiplicity : this means that there are some functions $\left(x, \xi^{\prime}\right) \mapsto \lambda_{i}\left(x, \xi^{\prime}\right)$, where $i=1, \ldots, d$, holomorphic in a neighbourhood of $\left(\bar{x}=0, \bar{\xi}^{\prime}=(1,0, \ldots, 0)\right)$ and some integers $m_{i} \geq 1$ such that

$$
g(x ; \xi)=\prod_{i=1}^{d}\left(\xi_{0}-\lambda_{i}\left(x, \xi^{\prime}\right)\right)^{m_{i}} \text { for }\left(x, \xi^{\prime}\right) \text { closed to }\left(\bar{x}, \bar{\xi}^{\prime}\right)
$$

and, if $\lambda_{i}=\lambda_{i}\left(\bar{x}, \bar{\xi}^{\prime}\right), \lambda_{i} \neq \lambda_{j}$ if $i \neq j$.

Then, we can solve the non-linear first order Cauchy problem

$$
\left\{\begin{array}{l}
D_{0} k_{i}(x)=\lambda_{i}\left(x, D^{\prime} k_{i}(x)\right) \\
k_{i}(x)=x_{1} \quad \text { for } x_{0}=0
\end{array}\right.
$$

where $D^{\prime} k_{i}(x)=\left(D_{1} k_{i}(x), \ldots, D_{n} k_{i}(x)\right)$. There is a unique solution of this problem in a neighbourhood of the origin. We have $D k_{i}(0)=\left(\lambda_{i}, 1,0, \ldots, 0\right)$; so the functions $k_{i}$ can be assumed defined and holomorphic on $\Omega_{0}$ and $D k_{i}(x) \neq 0$ for $x \in \Omega_{0}$. This allows us to define hypersurfaces $K_{i}=\left\{x \in \Omega_{0} ; k_{i}(x)=0\right\}$; if $T$ is the hyperplane $x_{0}=x_{1}=0$ in $S$, then we have $K_{i} \cap S=\Omega_{0} \cap T$ : this means that the $K_{i}$ are the characteristic hypersurfaces which go out from $T$.

Now, the Hamada-Leray-Wagschal's theorem is called back. The following problem is studied

$$
\left\{\begin{array}{l}
a(x, D) u(x)=\left.\sum_{i=1}^{d} v_{i}(t, x)\right|_{t=k_{i}(x)}, \\
D_{0}^{h} u(x)=\left.w_{h}(t, x)\right|_{t=x_{1}} \quad \text { for } x_{0}=0,0 \leq h<m .
\end{array}\right.
$$

$\mathcal{R}\left(\dot{D}_{\omega}\right)$ will be denote the universal covering of $\dot{D}_{\omega}=\{t \in \mathbf{C} ; 0<|t|<\omega\}$. Then, we have the following theorem $[3,4]$

Theorem 1.1. Let $\Omega \subset \Omega_{0}$ a simply connected open neighbourhood of the origin of $\mathbf{C}^{n+1}$, there is a simply connected open neighbourhood $\Omega^{\prime} \subset \Omega$ of the origin of $\mathbf{C}^{n+1}$ and $\omega_{0}>0$ such that: let $0<\omega \leq \omega_{0}, a \in S \cap \Omega^{\prime}$ such that $0<\left|a_{1}\right|<\omega, v_{i}$ and $w_{h}$ some holomorphic germs at $(t, x)=\left(a_{1}, a\right)$ which have a holomorphic extension on $\mathcal{R}\left(\dot{D}_{\omega}\right) \times \Omega$, then the solution of (1.1) could be written

$$
u(x)=\left.\sum_{i=1}^{d} u_{i}(t, x)\right|_{t=k_{i}(x)} \text { for } x \text { closed to } a
$$


where $u_{i}$ is a holomorphic germ at $\left(a_{1}, a\right)$ which have a holomorphic extension on $\mathcal{R}\left(\dot{D}_{\omega}\right) \times \Omega^{\prime}$.

The aim goal of this paper is to explicit the $u_{i}$ 's structure in function of $v_{i}$ and $w_{h}$. More precisely, if the germs $v_{i}$ and $w_{h}$ are finite dimensional determination's functions, then $u_{i}$ are finite dimensional determination's function too [1]. Using a structure's theorem of finite dimensional determination's functions in a punctured disk and technics of [1], the $u_{i}$ 's form will be precise.

\section{§2. Notations, Recall and Main Theorem}

Let $X$ a connected complex manifold and $E$ a complex Banach space. The complex vector space of holomorphic functions $f: X \rightarrow E$ will be written $\mathcal{H}(X ; E)$ and $\mathcal{O}_{a}=\mathcal{O}_{a}(X ; E)$ will be the complex vector space of holomorphic germs at point $a \in X$ with values in $E$.

If $u \in \mathcal{O}_{a}$ has an analytic continuation along a path $\gamma: I \rightarrow X$, where $I=[0,1]$, of origin $a$ and endpoint $b$, we will write $u_{\gamma} \in \mathcal{O}_{b}$ the germ in $b$ got by analytic continuation of $u$ along $\gamma$.

$\Gamma_{a}=\Gamma_{a}(X)$ will be the space of loops $\gamma: I \rightarrow X$ of origin $a$ and let $u \in \mathcal{O}_{a}$ a germ which has an analytic continuation along all loops $\gamma \in \Gamma_{a}$. We note $F_{a}^{u}$ the subvector space of $\mathcal{O}_{a}$ spanned by $\left(u_{\gamma}\right)_{\gamma \in \Gamma_{a}}$ of all determinations of $u$ at point $a$ and $A_{\gamma}^{u} \in G L\left(F_{a}^{u}\right)$ the automorphism

$$
A_{\gamma}^{u}: \theta \in F_{a}^{u} \longmapsto \theta_{\gamma} \in F_{a}^{u} .
$$

This automorphism depends only on the homotopy class $[\gamma]$ of the path $\gamma \in \Gamma_{a}$, then we have a linear representation of Poincaré's group $\pi_{1}(X, a)$

$$
A^{u}:[\gamma] \in \pi_{1}(X, a) \longmapsto A_{\gamma}^{u} \in G L\left(F_{a}^{u}\right) \text { where } \gamma \in[\gamma] ;
$$

this linear representation is called the monodromy of germ $u$.

The germ $u$ is of finite dimensional determination if $F_{a}^{u}$ is a finite dimensional vector space. The space $\mathcal{O}_{a}^{f}$ of germs of finite dimensional determination is a subvector space of $\mathcal{O}_{a}$. For $u \in \mathcal{O}_{a}^{f}, \sigma_{\gamma}(u)$ is the $A_{\gamma}^{u}$ automorphism's spectrum. When $u$ has a holomorphic extension on $X$, we have $u_{\gamma}=u$ for all $\gamma \in \Gamma_{a}$, so $F_{a}^{u}=\mathbf{C} u$ and $A_{\gamma}^{u}$ is the identity map. When $u$ is not the null germ, $\operatorname{dim} F_{a}^{u}=1$ and $\sigma_{\gamma}(u)=\{1\}$; when $u=0$, we will say that $\sigma_{\gamma}(u)=\emptyset$. At last, a subvector space $F$ of $\mathcal{O}_{a}$ is said invariant under analytic continuation if, for all $u \in F$ and $\gamma \in \Gamma_{a}, u$ has an analytic continuation along $\gamma$ and $u_{\gamma} \in F$.

Let $A=\left(a_{i, j}\right)_{i, j} \in M_{m, n}(\mathbf{C})$ with $1 \leq i \leq m$ and $1 \leq j \leq n$, then $i$ stands for the column and $j$ stands for the line. 
We call back the following structure's theorem. We note $\delta$ the loop defined by $s \mapsto\left(a_{1} e^{2 i \pi s}, a\right)$ where $\left(a_{1}, a\right) \in \dot{D}_{\omega} \times \Omega$. We have to remark that $\delta$ is a generator loop of $\pi_{1}\left(\dot{D}_{\omega} \times \Omega,\left(a_{1}, a\right)\right) \simeq \mathbf{Z}$.

Theorem 2.1. A germ $u \in \mathcal{O}_{\left(a_{1}, a\right)}\left(\mathcal{R}\left(\dot{D}_{\omega}\right) \times \Omega\right)$ is of finite dimensional determination if, and only if, it could be written

$$
u(t, x)=\sum_{m \in M} t^{p_{m}} \sum_{k=0}^{q_{m}} a_{m k}(t, x)[\ln t]^{k}
$$

where $M$ is a finite set, $\mathcal{R} e\left(p_{m}\right) \in\left[0,1\left[, p_{m} \neq p_{m^{\prime}}\right.\right.$ if $m \neq m^{\prime}$ and the functions $a_{m k}: \dot{D}_{\omega} \times \Omega \rightarrow \mathbf{C}$ are holomorphic, $a_{m q_{m}} \not \equiv 0$.

Moreover, $\left\{\lambda_{m}=e^{2 i \pi p_{m}}\right\}_{m \in M}$ are the eigenvalues of the automorphism $A_{\delta}^{u}$; to each eigenvalue $\lambda_{m}$, there is an unique Jordan's block of dimension $q_{m}+1$ and

$$
\operatorname{dim} F_{\left(a_{1}, a\right)}^{u}=\sum_{m \in M}\left(q_{m}+1\right)
$$

Now, let $u$ the following germ at point $\left(a_{1}, a\right)$

$$
u(t, x)=t^{p} \sum_{k=0}^{q} a_{k}(t, x)[\ln t]^{k}
$$

where functions $a_{k}: \dot{D}_{\omega} \times \Omega \rightarrow \mathbf{C}$ are holomorphic, $a_{q} \not \equiv 0$. This germ $u$ is of finite dimensional determination, $\operatorname{dim} F_{\left(a_{1}, a\right)}^{u}=q+1$ and $e^{2 i \pi p}$ is the only eigenvalue of the automorphism $A_{\delta}^{u}$. Moreover, it is easy to see that in all basis $\left(\psi_{0}, \ldots, \psi_{q}\right)$ of $F_{\left(a_{1}, a\right)}^{u}$, such that the matricial representation of $A_{\delta}^{u}$ in this basis is

$$
\left(\begin{array}{cccc}
e^{2 i \pi p} & 1 & & \\
& \ddots & \ddots & \\
& & e^{2 i \pi p} & 1 \\
& & & e^{2 i \pi p}
\end{array}\right),
$$

we have $\psi_{0}=C t^{p} a_{q}$ where $C \in \mathbf{C}^{*}$.

Now this is the main theorem.

First, some space called $h(p, q)$ are defined.

Definition 2.1. Let $p \in \mathbf{C}$ such that $\operatorname{Re} e(p) \in[0,1[$ and $q \in \mathbf{N} . u \in$ $h(p, q)$ if there is a real $\omega>0$ and a simply connected open neighbourhood $\Omega$ 
of the origin of $\mathbf{C}^{n+1}$ such that $u \in \mathcal{H}\left(\mathcal{R}\left(\dot{D}_{\omega}\right) \times \Omega\right)$ could be written under the form

$$
\sum_{k=0}^{q} t^{p} a_{k}(t, x)[\ln t]^{k}
$$

where the $a_{k}$ are holomorphic on $\dot{D}_{\omega} \times \Omega$ and where $a_{q} \in \mathcal{H}\left(D_{\omega} \times \Omega\right)$ when $p=0$ with $D_{\omega}=\{t \in \mathbf{C} ;|t|<\omega\}$.

Theorem 2.2. If the germs $v_{i}, w_{h}$ are in a same $h(p, q)$ space, then the solution $u$ of problem (1.1) could be written

$$
u(x)=\sum_{i=1}^{d} u_{i}\left(k_{i}(x), x\right)
$$

with $u_{i} \in h(p, q)$ for $i=1, \ldots, d$.

Remark. Let $u \in h(p, q)$ and $k$ a holomorphic function in a neighbourhood of the origin, then, for all holomorphic linear differential operator $a(x, D)$, we have

$$
a(x, D) u(k(x), x)=v(k(x), x)
$$

where $v \in h(p, q)$. This results from the fact that the $h(p, q)$ spaces are invariant under differentiation.

This remark allows us to assume the germs $w_{h} \equiv 0$, in Theorem 2.2.

We have to say that $\mathrm{C}$. Wagschal [5] gave an analogous result in the case of an operator with simple characteristics (that is $m_{i}=1$ for all $i$ and then $d=m)$. He defined $\tilde{h}(p, q)$ spaces.

Definition 2.2. Let $p \in \mathbf{C}$ and $q \in \mathbf{N} . u \in \tilde{h}(p, q)$ if there is a real $\omega>0$ and a simply connected open neighbourhood $\Omega$ of the origin of $\mathbf{C}^{n+1}$ such that $u \in \mathcal{H}\left(\mathcal{R}\left(\dot{D}_{\omega}\right) \times \Omega\right)$ could be written under the form

$$
\left\{\begin{array}{l}
t^{p} P_{q}(x,[\ln t]) \quad \text { if } p \text { is not a integer }<0 \\
t^{p} P_{q-1}(x,[\ln t])+P_{q}(x,[\ln t]) \quad \text { if } p \text { is an integer }<0 \text { and } q \geq 1 \\
P_{0}(x) \quad \text { if } p \text { is an integer }<0 \text { and if } q=0 .
\end{array}\right.
$$

where $P_{i}(x, \xi), \xi \in \mathbf{C}$ are polynomial functions in $\xi$ of degree $\leq i$, with holomorphic coefficients in a neighbourhood of the origin of $\mathbf{C}^{n+1}$. 
Then, he proved the following theorem [5]

Theorem 2.3. If the germs $v_{i}$ and $w_{h}$ are in a same $\tilde{h}(p, q)$ space, then the solution $u$ of problem (1.1) (in the case of an operator with simple characteristics) could be written

$$
u(x)=\sum_{i=1}^{d} u_{i}\left(k_{i}(x), x\right)
$$

with $u_{i} \in \tilde{h}(p, q)$ for $i=1, \ldots, d$.

Also, this theorem gives a result on the order of the polar singularity of the solution. This type of result could not be get for an operator with multiple characteristics of constant multiplicity. Indeed, Y. Hamada [2] gave the following very simple counterexample

$$
\left\{\begin{array}{l}
{\left[D_{0}^{2}-D_{1}\right] u(x)=0,} \\
u(x)=\frac{1}{x_{1}} \quad \text { for } x_{0}=0 \\
D_{0} u(x)=0 \quad \text { for } x_{0}=0 .
\end{array}\right.
$$

The solution is

$$
u(x)=\frac{1}{x_{1}}+\frac{1}{x_{1}} \sum_{n=1}^{\infty}(-1)^{n} \frac{n !}{(2 n) !}\left(\frac{x_{0}^{2}}{x_{1}}\right)^{n} ;
$$

then the solution $u$ have only essential singularities on the double characteristic hypersurface

$$
K=\left\{x ; x_{1}=0\right\}
$$

\section{§3. Recall on the Monodromy}

We come back to the problem (1.1)

$$
\left\{\begin{array}{l}
a(x, D) u(x)=\left.\sum_{i=1}^{d} v_{i}(t, x)\right|_{t=k_{i}(x)}, \\
D_{0}^{h} u(x)=w_{h}(t, x)_{t=x_{1}} \text { for } x_{0}=0,0 \leq h<m .
\end{array}\right.
$$

The solution of this problem could be written under the form

$$
u(x)=\left.\sum_{i=1}^{d} u_{i}(t, x)\right|_{t=k_{i}(x)}=\sum_{i=1}^{d}\left[D_{0}^{m-m_{i}} D_{t}^{m_{i}} U_{i}(t, x)\right]_{\mid t=k_{i}(x)}
$$


where $U_{i}$ is a germ at point $\left(a_{1}, a\right)$ which has a holomorphic extension on $\mathcal{R}\left(\dot{D}_{\omega}\right) \times \Omega^{\prime} . U=\left(U_{1}, \ldots, U_{d}\right)$ is the solution of an integro-differential problem

$$
\left\{\begin{aligned}
\left(D_{0}^{m}-A_{m}(x, D)\right) U(t, x) & =\sum_{l \in \mathcal{L}} A_{l}^{m}(x, D) D_{t}^{-l} U(t, x)+y_{m}(t, x) \\
\left(D_{0}^{h}-A_{h}(x, D)\right) U(t, x) & =\sum_{l \in \mathcal{L}} A_{l}^{h}(x, D) D_{t}^{-l} U(t, x)+y_{h}(t, x) \\
& \text { for } x_{0}=0,0 \leq h<m
\end{aligned}\right.
$$

with $\mathcal{L}$ a finite subset of $\mathbf{Z}^{*}$. The operators $A_{h}$ and $A_{l}^{h}$, for $h=0, \ldots, m$ and $l \in \mathcal{L}$, are holomorphic linear differential operators whose coefficients are holomorphic in $\Omega$. The assumptions about operators' orders are the following

$$
\begin{aligned}
& \operatorname{order} A_{h} \leq h, \quad \operatorname{order}_{x_{0}} A_{h}<h, \\
& \operatorname{order} A_{l}^{h} \leq \begin{cases}h+l-1 & \text { if } l<0, \\
h+l & \text { if } l>0 .\end{cases}
\end{aligned}
$$

Now, the functions $y_{h}, h=0, \ldots, m$ are defined.

$$
y_{m}(t, x)=\left(a_{1}(x) D_{t}^{-m} v_{1}(t, x), \ldots, a_{d}(x) D_{t}^{-m} v_{d}(t, x)\right)
$$

where $a_{i}$ are holomorphic functions on $\Omega$ and, for $0 \leq h \leq m-1$,

$$
y_{h}(t, x)=\left(\sum_{k=0}^{m-1} P_{1, k, h}\left(x^{\prime}, D_{t}^{-1}\right) w_{k}(t, x), \ldots, \sum_{k=0}^{m-1} P_{d, k, h}\left(x^{\prime}, D_{t}^{-1}\right) w_{k}(t, x)\right)
$$

where $P_{i, j, h}\left(x^{\prime}, \xi\right)$ is polynomial function in $\xi \in \mathbf{C}$ with holomorphic coefficients in the variable $x^{\prime}$. This kind of integro-differential problem was studied in [3, 4]. Section 4 of [1] shows that if the datas of problem (3.1) are of finite dimensional determination, then

$$
F_{\left(a_{1}, a\right)}^{U} \subset F
$$

where $F$ is a finite dimensional vector space invariant under analytic continuation. $F$ is spanned by the generators' system

$$
\left(S\left(Q_{\delta \pm 1} S\left(\beta_{j}\right)\right), S\left(\beta_{j}\right)\right)_{1 \leq j \leq q},
$$

with the following notations

- $\left(\beta_{j}\right)_{1 \leq j \leq q}$ is a basis of $F_{\left(a_{1}, a\right)}^{y}$, where $y=\left(y_{0}, \ldots, y_{m}\right)$,

- $S(y)$ is the solution of problem (3.1), 
- $\left(Q_{\delta^{ \pm 1}} u\right)_{h}=\sum_{l \in \mathcal{L}^{+}} A_{l}^{h}(x, D) P_{\delta^{ \pm 1}}^{l}(u), \mathcal{L}^{+}=\{l \in \mathcal{L} ; l>0\}$ and

$$
P_{\delta^{ \pm 1}}^{l}(u)=\left(D_{t}^{-l} u\right)_{\delta^{ \pm 1}}-D_{t}^{-l}\left(u_{\delta^{ \pm 1}}\right) .
$$

We can show that $P_{\delta^{ \pm 1}}^{l}(u)$ is holomorphic in a neighbourhood of the origin of $\mathbf{C}_{t} \times \mathbf{C}_{x}^{n+1}$, that $P_{\delta^{ \pm 1}}^{l}(u)=0$ if $u$ is holomorphic in a neighbourhood of the origin of $\mathbf{C}_{t} \times \mathbf{C}_{x}^{n+1}$ [1]. Moreover, if $y$ is holomorphic in a neighbourhood of the origin of $\mathbf{C}_{t} \times \mathbf{C}_{x}^{n+1}$, then so is $S(y)$ [4]. Hence $S\left(Q_{\delta^{ \pm 1}} S\left(\beta_{j}\right)\right)$ is holomorphic in a neighbourhood of the origin of $\mathbf{C}_{t} \times \mathbf{C}_{x}^{n+1}$.

These results allow us to show that a matricial representation of the automorphism $\theta \mapsto \theta_{\gamma}$, where $\gamma \in \Gamma_{\left(a_{1}, a\right)}\left(\dot{D}_{\omega} \times \Omega\right)$, in the generators' system (3.2) could be written

$$
\left(\begin{array}{ll}
I d & B \\
0 & M
\end{array}\right)
$$

where $M$ is the matricial representation of $A_{\gamma}^{y}$ in the basis $\left(\beta_{j}\right)_{1 \leq j \leq q}$.

At last, for all $i=1, \ldots, d$, thanks to the results of section 3 of [1], we know that there is a vector space $F_{i}$ of finite dimension which is invariant under analytic continuation such that

$$
F_{\left(a_{1}, a\right)}^{u_{i}} \subset F_{i}
$$

There is a generators' system of $F_{i}$ such that a matricial representation of the automorphism $\theta \mapsto \theta_{\gamma}$ in this generators' system is

$$
\left(\begin{array}{ll}
I d & B \\
0 & M
\end{array}\right) .
$$

Moreover, in section 5 of [1] the following fact was shown. If $1 \notin \sigma_{\delta}(y)$, then there is a finite dimensional vector space invariant under analytic continuation $\tilde{F}_{i}$, such that $F_{\left(a_{1}, a\right)}^{u_{i}} \subset \tilde{F}_{i}$, and a generators' system of $\tilde{F}_{i}$ such that a matricial representation of the automorphism $\theta \mapsto \theta_{\gamma}$ in this generators' system is $M$.

\section{$\S 4$. Proof of Theorem 2.2}

By linearity, we can assume $v_{1} \in h(p, q), v_{1} \neq 0$ and $v_{2}, \ldots, v_{d}=0$. 
So, the following problem is studied

$$
\left\{\begin{array}{l}
a(x, D) u(x)=\left.v_{1}(t, x)\right|_{t=k_{1}(x)}, \\
D_{0}^{h} u(x)=0 \quad \text { for } x_{0}=0,0 \leq h<m .
\end{array}\right.
$$

With help of previous section, the solution could be written under the form

$$
u(x)=\left.\sum_{i=1}^{d} u_{i}(t, x)\right|_{t=k_{i}(x)}=\sum_{i=1}^{d}\left[\left.D_{0}^{m-m_{i}} D_{t}^{m_{i}} U_{i}(t, x)\right|_{t=k_{i}(x)}\right.
$$

where $U_{i}$ is a germ at point $\left(a_{1}, a\right)$ which has a holomorphic extension on $\mathcal{R}\left(\dot{D}_{\omega}\right) \times \Omega^{\prime} . U=\left(U_{1}, \ldots, U_{d}\right)$ is solution of an integro-differential problem

$$
\left\{\begin{array}{c}
\left(D_{0}^{m}-A_{m}(x, D)\right) U(t, x)=\sum_{l \in \mathcal{L}} A_{l}^{m}(x, D) D_{t}^{-l} U(t, x)+y_{m}(t, x), \\
\left(D_{0}^{h}-A_{h}(x, D)\right) U(t, x) \\
=\sum_{l \in \mathcal{L}} A_{l}^{h}(x, D) D_{t}^{-l} U(t, x) \text { for } x_{0}=0,0 \leq h<m
\end{array}\right.
$$

with

$$
y_{m}(t, x)=\left(a(x) D_{t}^{-m} v_{1}(t, x), 0, \ldots, 0\right)
$$

where $a$ is a holomorphic function on $\Omega$.

With help of section 4 of [1], we know that $F_{\left(a_{1}, a\right)}^{U} \subset F$ where $F$ is a finite dimensional vector space invariant under analytic continuation. A generators' system of $F$ is given by

$$
\left(S\left(Q_{\delta^{ \pm 1}} S\left(\beta_{j}\right)\right), S\left(P_{\delta^{ \pm 1}}^{m}\left(D_{t}^{m} \beta_{j}\right)\right), S\left(\beta_{j}\right)\right)_{0 \leq j \leq k}
$$

where $\beta_{j}=\left[\left(a(x) D_{t}^{-m} \theta_{j}, 0 \ldots, 0\right), 0, \ldots, 0\right]$ and $\left(\theta_{j}\right)_{0 \leq j \leq k}$ is a basis of $F_{\left(a_{1}, a\right)}^{v_{1}}$. Indeed, the vector space spanned by $\left(P_{\delta^{ \pm 1}}^{m}\left(D_{t}^{m} \beta_{j}\right), \beta_{j}\right)_{0 \leq j \leq k}$ is invariant under analytic continuation and contains $F_{\left(a_{1}, a\right)}^{y}\left(y=\left(y_{m}, 0, \ldots, 0\right)\right)$. Moreover, since $P_{\delta^{ \pm 1}}^{m}\left(D_{t}^{m} \beta_{j}\right) \in \mathcal{H}\left(D_{\omega} \times \Omega^{\prime}\right)$, we have $Q_{\delta} P_{\delta^{ \pm 1}}^{m}\left(D_{t}^{m} \beta_{j}\right) \equiv Q_{\delta^{-1}} P_{\delta^{ \pm 1}}^{m}\left(D_{t}^{m} \beta_{j}\right) \equiv 0$; thus we can conclude with help of Section 3 .

Remark. We can show that $F$ is spanned by

$$
\left(S\left(Q_{\delta} S\left(\beta_{j}\right)\right), S\left(P_{\delta}^{m}\left(D_{t}^{m} \beta_{j}\right)\right), S\left(\beta_{j}\right)\right)_{0 \leq j \leq k} .
$$


Indeed, thanks to lemma 3.4 of [1], we get

$$
P_{e}^{l}(u)=P_{\delta^{-1} \delta}(u)=P_{\delta^{-1}}^{l}(u)+P_{\delta}^{l}\left(u_{\delta^{-1}}\right) ;
$$

but $P_{e}^{l}(u)=0$, so $P_{\delta^{-1}}^{l}(u)=-P_{\delta}^{l}\left(u_{\delta^{-1}}\right)$. Then we have

$$
P_{\delta^{-1}}^{m}\left(D_{t}^{m} \beta_{j}\right)=-P_{\delta}^{m}\left(\left(D_{t}^{m} \beta_{j}\right)_{\delta^{-1}}\right) .
$$

But the vector space spanned by $\left(D_{t}^{m} \beta_{j}\right)_{0 \leq j \leq k}$ is invariant under analytic continuation (because $\left.D_{t}^{m} \beta_{j}=\left[\left(a \theta_{j}, 0 \ldots, 0\right), 0, \ldots, 0\right]\right)$, so $P_{\delta^{-1}}^{m}\left(D_{t}^{m} \beta_{j}\right)$ lies in the vector space spanned by

$$
\left(S\left(Q_{\delta^{ \pm 1}} S\left(\beta_{j}\right)\right), S\left(P_{\delta}^{m}\left(D_{t}^{m} \beta_{j}\right)\right), S\left(\beta_{j}\right)\right)_{0 \leq j \leq k} .
$$

Now, from definition of operators $Q_{\gamma}$ and from the fact that $S(u)_{\gamma}=S\left(u_{\gamma}\right)+$ $S\left(Q_{\gamma} S(u)\right)$, we have

$$
\begin{aligned}
Q_{\delta^{-1}} S\left(\beta_{j}\right) & =-Q_{\delta}\left(\left(S\left(\beta_{j}\right)\right)_{\delta^{-1}}\right) \\
& =-Q_{\delta} S\left(\left(\beta_{j}\right)_{\delta^{-1}}\right)-Q_{\delta}\left(S\left(Q_{\delta^{-1}}\left(\beta_{j}\right)\right)\right) \\
& =-Q_{\delta} S\left(\left(\beta_{j}\right)_{\delta^{-1}}\right)
\end{aligned}
$$

because $S\left(Q_{\delta^{-1}} S\left(\beta_{j}\right)\right) \in \mathcal{H}\left(D_{\omega} \times \Omega^{\prime}\right)$. Now, since the vector space spanned by $\left(P_{\delta}^{m}\left(D_{t}^{m} \beta_{j}\right), \beta_{j}\right)_{0 \leq j \leq k}$ is invariant under analytic continuation and since $P_{\delta}^{m}\left(D_{t}^{m} \beta_{j}\right)$ is holomorphic on $D_{\omega} \times \Omega^{\prime}, Q_{\delta} S\left(\left(\beta_{j}\right)_{\delta^{-1}}\right)$ lies in the vector space spanned by

$$
\left(S\left(Q_{\delta} S\left(\beta_{j}\right)\right), S\left(P_{\delta}^{m}\left(D_{t}^{m} \beta_{j}\right)\right), S\left(\beta_{j}\right)\right)_{0 \leq j \leq k} .
$$

Hence, $F$ is spanned by the generators' system (4.1)

First, we look to the case $p \neq 0$.

There is a basis of $F_{\left(a_{1}, a\right)}^{v_{1}}$ such that the matricial representation of $A_{\delta}^{v_{1}}$ in this basis is

$$
M=\left(\begin{array}{cccc}
e^{2 i \pi p} & 1 & & \\
& \ddots & \ddots & \\
& & e^{2 i \pi p} & 1 \\
& & & e^{2 i \pi p}
\end{array}\right) .
$$

So, by Section 3, we know that the solution of problem (1.1) is of finite dimensional determination and could be written under the form

$$
u(x)=\sum_{i=1}^{d} u_{i}\left(k_{i}(x), x\right) .
$$


Moreover, always by Section 3 , since $1 \notin \sigma\left(v_{1}\right)$, there exists a finite dimensional vector space $\tilde{F}_{i}$ invariant under analytic continuation which contains $F_{\left(a_{1}, a\right)}^{u_{i}}$ and such that $M$ is a matricial representation of the automorphism $\theta \mapsto \theta_{\delta}$ in a generators' system of $\tilde{F}_{i}$.

The end of the proof use corollary 3.1 of [1] which is recalled.

Corollary 4.1. Let $F$ a finite dimensional vector space, $A \in \mathcal{L}(F)$ a homomorphism whose a matricial representation in a generators' system is given by a matrix $M$. Then, we have

$$
\sigma(A) \subset \sigma(M)
$$

where $\sigma(A)$ is the set of eigenvalues of $A$ and $\sigma(M)$ is the set of eigenvalues of $M$.

Hence, with help of Theorem 2.1 and Corollary 4.1, we deduce that $u_{i} \in$ $h(p, q)$ for $p \neq 0$.

Now, we look to the case $p=0$.

We have

$$
v_{1}(t, x)=\sum_{k=0}^{q} a_{k}(t, x)[\ln t]^{k}
$$

with $a_{q} \in \mathcal{H}\left(D_{\omega} \times \Omega\right)$. By linearity, two cases will be studied:

1. $v_{1}(t, x)=a_{k}(t, x)[\ln t]^{k}$ with $0 \leq k \leq q-1$,

2. $v_{1}(t, x)=a_{q}(t, x)[\ln t]^{q}$.

First case - Let $\left(\theta_{0}, \ldots, \theta_{k}\right)$ a basis of $F_{\left(a_{1}, a\right)}^{v_{1}}$ such that the matricial representation of the automorphism $A_{\delta}^{v_{1}}$, in this basis, is

$$
M_{k+1}=\left(\begin{array}{ccccc}
1 & 1 & & \\
& \ddots & \ddots & \\
& & 1 & 1 \\
& & & 1
\end{array}\right) .
$$

We recall that $\theta_{0}=K a_{k}$ where $K \in \mathbf{C}^{*}$.

A matricial representation of $\theta \mapsto \theta_{\delta}$ in the generators' system (4.1) is given by

$$
\left(\begin{array}{lr}
I d_{2 k+2} & B \\
0 & M_{k+1}
\end{array}\right)
$$


where

$$
B=\left(\begin{array}{l}
I d_{k+1} \\
I d_{k+1}
\end{array}\right)
$$

and $I d_{j}$ is the identity matrix of dimension $j$.

Indeed, we have

- $S\left(Q_{\delta} S\left(\beta_{j}\right)\right)_{\delta}=S\left(Q_{\delta} S\left(\beta_{j}\right)\right)$ because $S\left(Q_{\delta} S\left(\beta_{j}\right)\right) \in \mathcal{H}\left(D_{\omega} \times \Omega^{\prime}\right)$,

- $S\left(P_{\delta}^{m}\left(D_{t}^{m} \beta_{j}\right)\right)_{\delta}=S\left(P_{\delta}^{m}\left(D_{t}^{m} \beta_{j}\right)\right)$ because $S\left(P_{\delta}^{m}\left(D_{t}^{m} \beta_{j}\right)\right) \in \mathcal{H}\left(D_{\omega} \times \Omega^{\prime}\right)$,

- $S\left(\beta_{j}\right)_{\delta}=S\left(\beta_{j \delta}\right)+S\left(Q_{\delta} S\left(\beta_{j}\right)\right)$ and $\beta_{j \delta}=P_{\delta}^{m}\left(D_{t}^{m} \beta_{j}\right)+\beta_{j}+\beta_{j-1}$ with $\beta_{-1} \equiv 0$.

The matrix (4.2) could be written in an another basis, under the following Jordan's form

$$
\left(\begin{array}{lr}
I d_{2 k+1} & 0 \\
0 & M_{k+2}
\end{array}\right)
$$

the matrix of change of basis is

$$
\left(\begin{array}{lr}
C & D \\
0 & I d_{k+1}
\end{array}\right) .
$$

Using the matrix of change of basis (4.4), we define $\varphi_{0}, \ldots, \varphi_{2 k}, \psi_{0}, \ldots, \psi_{k+1}$ like this

$$
\begin{aligned}
& \varphi_{j}=\sum_{i=0}^{k}\left(c_{i, j} S\left(Q_{\delta} S\left(\beta_{i}\right)\right)+c_{i+k+1, j} S\left(P_{\delta}^{m}\left(D_{t}^{m} \beta_{i}\right)\right)\right) \quad \text { for } j=0, \ldots, 2 k \\
& \psi_{0}=\sum_{i=0}^{k}\left(c_{i, 2 k+1} S\left(Q_{\delta} S\left(\beta_{i}\right)\right)+c_{i+k+1,2 k+1} S\left(P_{\delta}^{m}\left(D_{t}^{m} \beta_{i}\right)\right)\right) \\
& \psi_{j}=\sum_{i=0}^{k}\left(d_{i, j-1} S\left(Q_{\delta} S\left(\beta_{i}\right)\right)+d_{i+k+1, j-1} S\left(P_{\delta}^{m}\left(D_{t}^{m} \beta_{i}\right)\right)\right)+S\left(\beta_{j-1}\right) \\
& \text { for } j=1, \ldots, k+1 .
\end{aligned}
$$

where the matrix $C$ is denoted by $C=\left(c_{i, j}\right)_{0 \leq i, j \leq 2 k+1}$ and $D$ is denoted by $D=\left(d_{i, j}\right)_{i, j}$ with $0 \leq i \leq 2 k+1$ and $0 \leq j \leq k$. We can note that $\varphi_{0}, \ldots, \varphi_{2 k}$ and $\psi_{0}$ are holomorphic on $D_{\omega} \times \Omega^{\prime}$. $F$ is spanned by $\left(\varphi_{0}, \ldots, \varphi_{2 k}, \psi_{0}, \ldots, \psi_{k+1}\right)$ since (4.4) is a matrix of change of basis and a matricial representation of the automorphism $\theta \mapsto \theta_{\delta}$ in this generators' system is given by (4.3). 
The following lemma is fundamental to end the proof of Theorem 2.2.

Lemma 4.1. $\quad$ Let $F$ a finite dimensional vector space invariant under analytic continuation such that $F_{\left(a_{1}, a\right)}^{U} \subset F$. Assume that $F$ is spanned by a generators' system such that a matricial representation $\theta \mapsto \theta_{\delta}$, in this generators' system, could be written

$$
\left(\begin{array}{lr}
I d & 0 \\
0 & M
\end{array}\right)
$$

where $M$ is a Jordan's matrix and 1 is an eigenvalue of $M$. Then, the solution of problem (1.1) could be written under the form

$$
\left.\sum_{i=1}^{d} u_{i}(t, x)\right|_{t=k_{i}(x)}
$$

with $F_{\left(a_{1}, a\right)}^{u_{i}} \subset F_{i}$ where $F_{i}$ is a finite dimensional vector space invariant under analytic continuation such that a matricial representation of the automorphism $\theta \mapsto \theta_{\delta}$ of $F_{i}$ in a generators' system is $M$.

Proof. We note $M=\operatorname{diag}\left(J_{\lambda_{1}}, \ldots, J_{\lambda_{n}}\right)$ where $J_{\lambda_{j}}$ is a Jordan's matrix with unique eigenvalues $\lambda_{j}$. We assume $\lambda_{1}=1$. Also, we note $\left(\varphi_{k}, \psi_{l}\right)_{k l}$, where $\varphi_{k} \in \mathcal{H}\left(\dot{D}_{\omega} \times \Omega^{\prime}\right), \psi_{l} \in \mathcal{H}\left(\mathcal{R}\left(\dot{D}_{\omega}\right) \times \Omega^{\prime}\right)$, a generators' system of $F$ such that a matricial representation of $\theta \mapsto \theta_{\delta}$ in this generators' system is

$$
\left(\begin{array}{lr}
I d & 0 \\
0 & M
\end{array}\right) .
$$

We have, for all $i=1, \ldots, d$,

$$
U_{i}(t, x)=\sum_{k} \nu_{k} \varphi_{k}^{i}+\sum_{l} \mu_{l} \psi_{l}^{i}
$$

We note $\varphi=\left(\sum_{k} \nu_{k} \varphi_{k}^{i}\right)_{1 \leq i \leq d}$ and $\left(\psi_{0}, \ldots, \psi_{l^{\prime}}\right)$ the generators' system associated to the Jordan's block $J_{1}$.

First, if, for $l=1, \ldots, l^{\prime}, \mu_{l}=0$, then we note $\tilde{\psi}_{0}=\varphi$ and we add to $\tilde{\psi}_{0}$ some vectors $\tilde{\psi}_{1}, \ldots, \tilde{\psi}_{l^{\prime}}$ to form a vector space invariant under analytic continuation such that a matricial representation of the automorphism $\theta \mapsto \theta_{\delta}$, in the generators' system, $\left(\tilde{\psi}_{0}, \ldots, \tilde{\psi}_{l^{\prime}}\right)$ is $J_{1}$. We note, for $l>l^{\prime}, \tilde{\psi}_{l}=\psi_{l}$. Then, we have

$$
U_{i}=\sum_{l} \mu_{l}^{\prime} \tilde{\psi}_{l}^{i}
$$


So, we have $F_{\left(a_{1}, a\right)}^{U_{i}} \subset G_{i}$ where $G_{i}$ is the vector space invariant under analytic continuation spanned by $\left(\tilde{\psi}_{l}\right)_{l}$ such that a matricial representation of the automorphism $\theta \mapsto \theta_{\delta}$, in the generators' system $\left(\tilde{\psi}_{l}\right)_{l}$, is $M$. Hence, by virtue of $u_{i}=D_{0}^{m-m_{i}} D_{t}^{m_{i}} U_{i}$, we have $F_{\left(a_{1}, a\right)}^{u_{i}} \subset F_{i}$ where $F_{i}$ is a vector space invariant under analytic continuation such that a matricial representation of the automorphism $\theta \mapsto \theta_{\delta}$ is $M$. We have to note that if $\varphi \in \mathcal{H}\left(D_{\omega} \times \Omega^{\prime}\right)$ then, by construction so is $\tilde{\psi}_{0}$.

Next, we note $l_{0}=\max \left\{l=0, \ldots, l^{\prime} ; \mu_{l} \neq 0\right\}$. So, we note

$$
\left\{\begin{array}{l}
\tilde{\psi}_{l}=\psi_{l} \quad \text { if } l>l^{\prime}, \\
\tilde{\psi}_{l}=\psi_{l} \quad \text { if } l<l_{0}, \\
\tilde{\psi}_{l_{0}}=\psi_{l_{0}}+\frac{1}{\mu_{l_{0}}} \varphi
\end{array}\right.
$$

and we add to $\left(\tilde{\psi}_{0}, \ldots, \tilde{\psi}_{l_{0}}\right)$ some vectors $\tilde{\psi}_{l_{0}+1}, \ldots, \tilde{\psi}_{l^{\prime}}$ to form a vector space invariant under analytic continuation such that a matricial representation of the automorphism $\theta \mapsto \theta_{\delta}$ in the generators' system $\left(\tilde{\psi}_{0}, \ldots, \tilde{\psi}_{l^{\prime}}\right)$ is $J_{1}$. We have to note, in this case, that if $\psi_{0} \in \mathcal{H}\left(D_{\omega} \times \Omega^{\prime}\right)$ and $\varphi \in \mathcal{H}\left(D_{\omega} \times \Omega^{\prime}\right)$ if $l_{0}=0$ then, by construction, $\tilde{\psi}_{0} \in \mathcal{H}\left(D_{\omega} \times \Omega^{\prime}\right)$.

Then, we have

$$
U_{i}=\sum_{l} \mu_{l} \tilde{\psi}_{l}^{i}
$$

So, we have $F_{\left(a_{1}, a\right)}^{U_{i}} \subset G_{i}$ where $G_{i}$ is the vector space invariant under analytic continuation spanned by $\left(\tilde{\psi}_{l}\right)_{l}$ such that a matricial representation of the automorphism $\theta \mapsto \theta_{\delta}$, in the generators' system $\left(\tilde{\psi}_{l}\right)_{l}$, is $M$. Hence, by virtue of $u_{i}=D_{0}^{m-m_{i}} D_{t}^{m_{i}} U_{i}$, we have $F_{\left(a_{1}, a\right)}^{u_{i}} \subset F_{i}$ where $F_{i}$ is a vector space invariant under analytic continuation such that a matricial representation of the automorphism $\theta \mapsto \theta_{\delta}$ in the generators' system $\left(D_{0}^{m-m_{i}} D_{t}^{m_{i}} \tilde{\psi}_{l}^{i}\right)_{l}$ is $M$. This is end the proof of the lemma.

The matrix (4.3) is a Jordan's matrix; so, with help of Lemma 4.1, we could write

$$
u(x)=\sum_{i=1}^{d} u_{i}\left(k_{i}(x), x\right)
$$

with $F_{\left(a_{1}, a\right)}^{u_{i}} \subset F_{i}$ where $F_{i}$ is a vector space invariant under analytic continuation and such that a matricial representation in a generators' system is given 
by $M_{k+2}$. Hence, with help of Theorem 2.1 and Corollary 4.1, we have

$$
u_{i}(t, x)=\sum_{j=0}^{k+1} a_{j}(t, x)[\ln t]^{j}
$$

where $a_{j} \in \mathcal{H}\left(\dot{D}_{\omega} \times \Omega^{\prime}\right)$ and $a_{k+1}=K_{i} D_{0}^{m-m_{i}} D_{t}^{m_{i}} \tilde{\psi}_{0}^{i}$ where $\tilde{\psi}_{0}$ is given in the proof of Lemma 4.1. By construction, we have $\tilde{\psi}_{0} \in \mathcal{H}\left(D_{\omega} \times \Omega^{\prime}\right)$ because $\varphi_{0}, \ldots, \varphi_{2 k}$ and $\psi_{0}$ are holomorphic on $D_{\omega} \times \Omega^{\prime}\left(\tilde{\psi}_{0}\right.$ is eventually null). Hence $u_{i} \in h(0, k+1)$.

Second case - As previously, a generators' system of the vector space $\tilde{F}$ is given by

$$
\left(S\left(Q_{\delta} S\left(\beta_{l}\right)\right), S\left(P_{\delta}^{m}\left(D_{t}^{m} \beta_{l}\right)\right), S\left(\beta_{l}\right)\right)_{0 \leq l \leq q}
$$

where $\beta_{l}=\left(a(x) D_{t}^{-m} \theta_{l}, 0 \ldots, 0\right)$ with $a \in \mathcal{H}(\Omega)$.

We have to remark that, since $\theta_{0} \in \mathcal{H}\left(D_{\omega} \times \Omega\right)$, we have $S\left(\beta_{0}\right) \in \mathcal{H}\left(D_{\omega} \times \Omega^{\prime}\right)$ and then $S\left(Q_{\delta} S\left(\beta_{0}\right)\right)=S\left(P_{\delta}^{m}\left(D_{t}^{m} \beta_{0}\right)\right)=0$.

Then, a generators' system of $F$ is given by

$$
\left(S\left(Q_{\delta} S\left(\beta_{l}\right)\right), S\left(P_{\delta}^{m}\left(D_{t}^{m} \beta_{l}\right)\right), S\left(\beta_{0}\right), S\left(\beta_{l}\right)\right)_{1 \leq l \leq q}
$$

and a matricial representation of the automorphism $\theta \mapsto \theta_{\delta}$ in this generators' system is given by

$$
\left(\begin{array}{lr}
I d_{2 q} & B^{\prime} \\
0 & M_{q+1}
\end{array}\right)
$$

where

$$
B^{\prime}=\left(\begin{array}{lllll}
0 & 1 & & \\
& & \ddots & \ddots & \\
& & & 0 & 1 \\
0 & 1 & & \\
& \ddots & \ddots & \\
& & & 0 & 1
\end{array}\right) .
$$

This follows from the same fact as above and $S\left(\beta_{0}\right) \in \mathcal{H}\left(D_{\omega} \times \Omega^{\prime}\right)$.

Now, (4.5) could be written in an another basis under the form

$$
\left(\begin{array}{lr}
I d_{2 q} & 0 \\
0 & M_{q+1}
\end{array}\right) .
$$


The matrix of change of basis is

$$
\left(\begin{array}{lr}
C & D \\
E & I d_{q+1}
\end{array}\right)
$$

where

$$
E=\left(\begin{array}{ccc}
e_{1} & \ldots & e_{2 q} \\
0 & \ldots & 0 \\
\vdots & & \vdots \\
0 & \ldots & 0
\end{array}\right)
$$

Using the matrix of change of basis (4.7), we define $\varphi_{1}, \ldots, \varphi_{2 q}, \psi_{0}, \ldots, \psi_{q}$ like this

$$
\begin{gathered}
\varphi_{j}=\sum_{i=1}^{q}\left(c_{i, j} S\left(Q_{\delta} S\left(\beta_{i}\right)\right)+c_{i+q, j} S\left(P_{\delta}^{m}\left(D_{t}^{m} \beta_{i}\right)\right)\right)+e_{j} S\left(\beta_{0}\right) \\
\text { for } j=1, \ldots, 2 q, \\
\psi_{j}=\sum_{i=1}^{q}\left(d_{i, j+1} S\left(Q_{\delta} S\left(\beta_{i}\right)\right)+d_{i+q, j+1} S\left(P_{\delta}^{m}\left(D_{t}^{m} \beta_{i}\right)\right)\right)+S\left(\beta_{j}\right) \\
\text { for } j=0, \ldots, q,
\end{gathered}
$$

where the matrix $C$ is denoted by $C=\left(c_{i, j}\right)_{1 \leq i, j \leq 2 q}$ and $D$ is denoted by $D=\left(d_{i, j}\right)_{i, j}$ with $1 \leq i \leq q+1$ and $1 \leq j \leq 2 q$. We can note that $\varphi_{1}, \ldots, \varphi_{2 q}$ and $\psi_{0}$ are holomorphic on $D_{\omega} \times \Omega^{\prime}$. $F$ is spanned by $\left(\varphi_{1}, \ldots, \varphi_{2 q}, \psi_{0}, \ldots, \psi_{q}\right)$ since (4.7) is a matrix of change of basis and a matricial representation of the automorphism $\theta \mapsto \theta_{\delta}$ in this generators' system is given by (4.6). So with help of Lemma 4.1, we could write

$$
u(x)=\sum_{i=1}^{d} u_{i}\left(k_{i}(x), x\right)
$$

with $F_{\left(a_{1}, a\right)}^{u_{i}} \subset F_{i}$ where $F_{i}$ is a vector space invariant under analytic continuation and such that a matricial representation in a generators' system is given by $M_{q+1}$. So, with help of Theorem 2.1 and Corollary 4.1, we have

$$
u_{i}(t, x)=\sum_{j=0}^{q} a_{j}(t, x)[\ln t]^{j}
$$

where $a_{j} \in \mathcal{H}\left(\dot{D}_{\omega} \times \Omega^{\prime}\right)$ and $a_{q}=K_{i} D_{0}^{m-m_{i}} D_{t}^{m_{i}} \tilde{\psi}_{0}^{i}$ where $\tilde{\psi}_{0}$ is given in the proof of Lemma 4.1. By construction, we have $\tilde{\psi}_{0} \in \mathcal{H}\left(D_{\omega} \times \Omega^{\prime}\right)$ because 
$\varphi_{1}, \ldots, \varphi_{2 q}$ and $\psi_{0}$ are holomorphic on $D_{\omega} \times \Omega^{\prime}$. Hence $u_{i} \in h(0, q)$. This is end the proof of Theorem 2.2 .

\section{References}

[1] Camalès, R., Sur la monodromie du problème de Cauchy ramifié, J. Math. Pures Appl., 81 (2002), 603-640.

[2] Hamada, Y., On the propagation of singularities of the solution of the Cauchy problem, Publ. RIMS, Kyoto Univ., 6 (1970), 357-384.

[3] Hamada, Y., Leray, J. and Wagschal, C., Systèmes d'équations aux dérivées partielles à caractéristiques multiples: problème de Cauchy ramifié; hyperbolicité partielle, J. Math. Pures Appl., 55 (1976), 297-352.

[4] Pongérard, P. and Wagschal, C., Ramification non abélienne, J. Math. Pures Appl., 77 (1998), 51-88.

[5] Wagschal, C., Problème de Cauchy analytique à données méromorphes, J. Math. Pures Appl., 51 (1972), 375-397. 